\title{
The In-Situ Resource Utilization Project under the New Exploration Enterprise
}

\author{
William E. Larson ${ }^{1}$ \\ NASA, Kennedy Space Center, FL, 32899 \\ and \\ Gerald B. Sanders ${ }^{2}$ \\ NASA, Johnson Space Center, Houston, TX, 77058
}

\section{Abstract}

The In Situ Resource Utilization Project under the Exploration Technology Development Program has been investing in technologies to produce Oxygen from the regolith of the moon for the last few years. Much of this work was demonstrated in a lunar analog field demonstration in February of 2010 . This paper will provide an overview of the key technologies demonstrated at the field demonstration will be discussed along with the changes expected in the ISRU project as a result of the new vision for Space Exploration proposed by the President and enacted by the Congress in the NASA Authorization Act of 2010.

\section{Introduction}

Tust a few weeks after the Aerospace Sciences Meeting in January of 2010, President Obama proposed a significant change in United States Space Policy. The new policy turned away from the Constellation Program and its focus to build a lunar outpost in the early 2020 's. Instead, the policy calls for an incremental approach to exploration that expands our exploration beyond earth orbit, visiting Asteroids as well as the Moon and ultimately leads to the surface of Mars. The policy also increased investment in technologies that will enable human exploration of the solar system as well as enhance the competitiveness of the Nation. The In-Situ Resource Utilization (ISRU) project stands to benefit from this increased technology investment. ISRU is one of the technologies that NASA was specifically directed to pursue by the Office of Management and Budget when the budget was released on February $1 \mathrm{st}, 2010$ and it is also specifically called out in the NASA Authorization Bill.

For the last several years, the ISRU project has been investing exclusively on lunar technologies through Constellation's Exploration Technology Development Program (ETDP). Under Constellation, ISRU has made significant strides in the area of oxygen production from lunar regolith. ISRU is no longer a laboratory experiment. It has been taken into the field to be tested end-to-end at lunar analogs and integrated with many other technology programs. The 2010 field demonstration was particularly successful and will be described below. In spite of this great progress the project was always constrained by funding under ETDP as budget problems within the Agency and within Constellation bled money away from the technology program. This also caused the project to become more and more of an in-house activity as NASA tried to maintain our core competencies. There is hope that this will change in the coming years.

The ISRU project under the new Space Policy will be quite different from the current project. It is a multidestination project now with a very broad scope of technology needs. Additionally, the project will now move beyond terrestrial analog demonstration to actual flight demonstrations on robotic precursor missions. The demonstration missions may be NASA sponsored or they may take advantage of Google X-Prize Landers or even share a ride on an International mission. Regardless of the means, it is the ISRU project's intent to fly missions to both the moon and Mars within this decade and also potentially to Near-Earth Asteroids.

\footnotetext{
${ }^{1}$ ISRU Project Manager, Engineering \& Technology Directorate, NE-I2, Member.
}

2 ISRU Deputy Project Manager, Energy Systems Division, EP, Member.

1 
III. 2010 ISRU Project Field Demonstration

The ISRU Field Demonstration on the slopes of the Hawaiian volcano Mauna Kea far exceeded the complexity of our earlier field tests. In 2010 , the demonstration was truly a "dust to thrust" endeavor. That is we had all the systems in place for an end-to-end demonstration of the ISRU process, from excavation of the dust (volcanic tephra that has similar characteristics to lunar regolith), through the utilization of the oxygen produced to provide thrust through an oxygen-methane thruster. Figure 1 shows the elements that the project had in place on Mauna Kea.

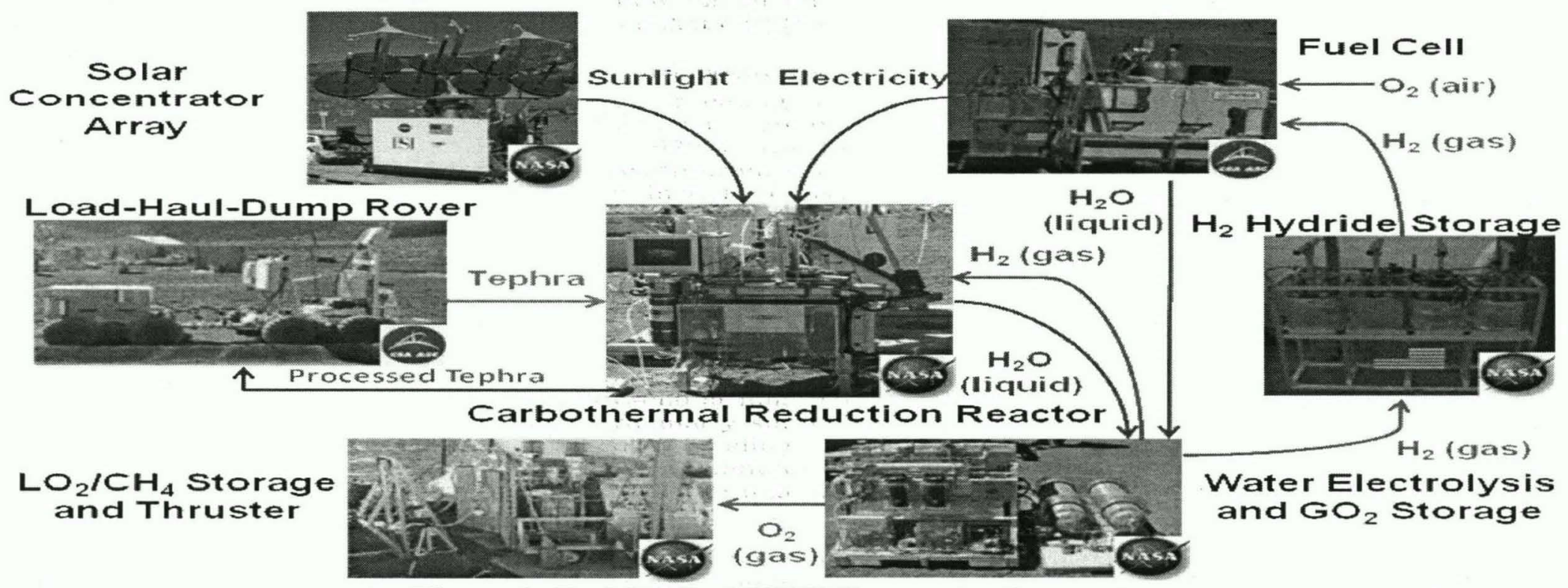

Figure 1: End-To-End ISRU Demonstration

Many of these elements were initially funded by NASA's Small Business Innovative Research Program (SBIR) and represents the type of success this program can yield. Other elements were contributed by the Canadian Space Agency and demonstrates the value of international partners. The only thing that was lacking from this demonstration that would have completely closed the fluids \& power loop is a photovoltaic array needed to power the electrolyzer. All other elements derived their power from the sun or the fuel cell.

\section{A. Oxygen Production}

The process of oxygen production starts with the acquisition of the raw material from which the oxygen is extracted. Our field test site was selected primarily because it had volcanic soil that has similarities to the lunar regolith. The test site on the slopes of Mauna Kea is rich in a volcanic material called Tephra. Tephra is essentially a fine ash product that is thrown into the air by the volcano. It collected in the valley area we selected for testing and is rich in reducible oxides and had a particle size distribution that is also similar to lunar regolith. The harvest the tephra needed for our tests, the Canadian Space Agency, through its contractor the Northern Centre for Advanced Technology (NORCAT), provided a small rover with a scoop similar to a front end loader. The rover collected a scoop full of tephra and delivered it to a feed system, where it was transported into the reactor. The feed system used this year was based on SBIR work performed by Honeybee in partnership with NASA. The feed system uses gas to move the tephra from the feed hopper at ground level up into the feed hopper on the reactor a meter or so off the ground. This system not only moves the tephra efficiently, it also helps with size sorting the as a cyclone separator is used at the top to separate the material from the gas stream. Both the rover and the feed transfer system performed flawlessly throughout the field demonstration. 


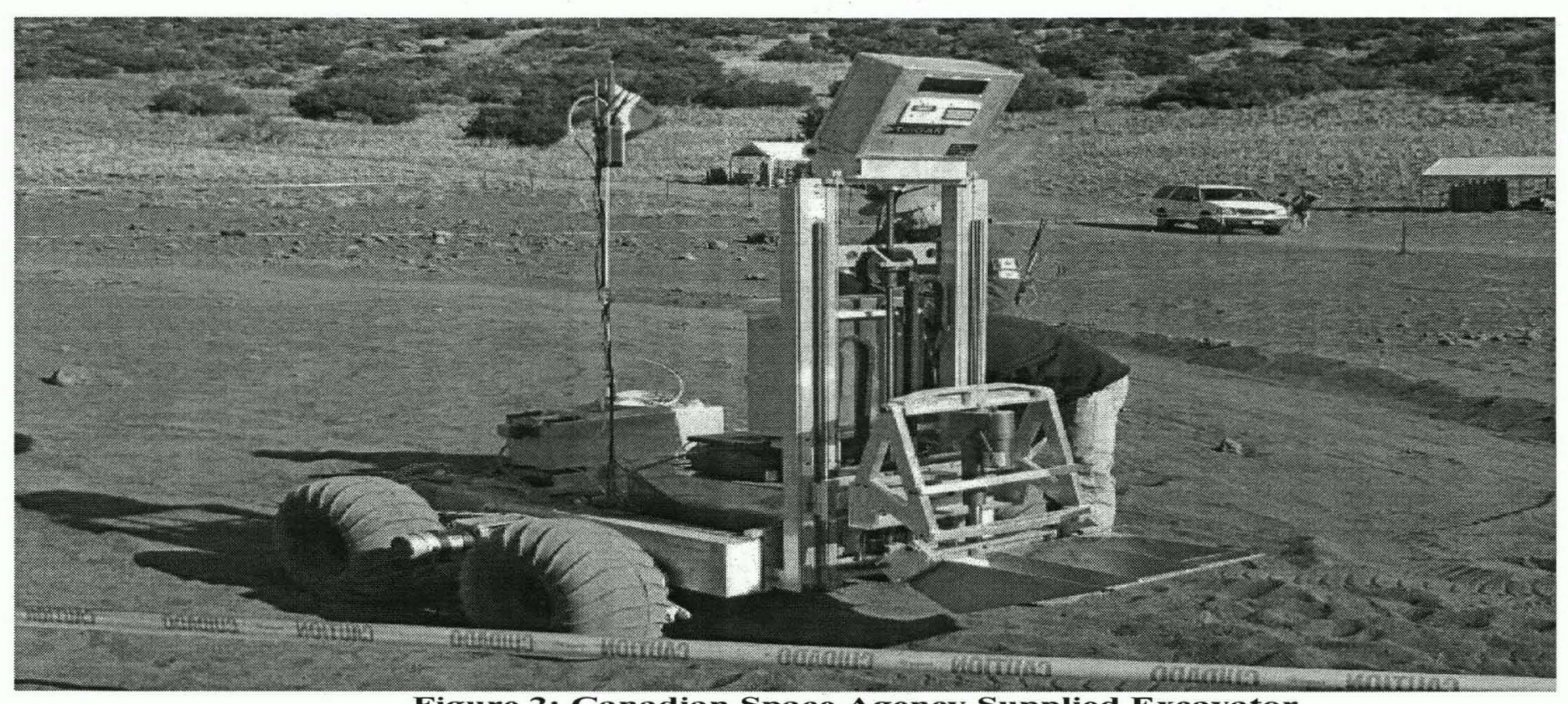

Figure 2: Canadian Space Agency Supplied Excavator

The ISRU project took a Carbothermal Reduction Process oxygen production system to the field for our 2010 tests. The Carbothermal system was the second of the ISRU project's oxygen production processes to be field tested. In the 2008 field demonstration two Hydrogen Reduction reactors were tested. Hydrogen reduction is the simplest method of producing oxygen from the lunar regolith, but it has limited oxygen yield because it can only reduce a small fraction of the lunar minerals. Carbothermal, on the other hand, has the ability to reduce many of the silicates in the regolith where a significant percentage oxygen is bound.

The Carbothermal reaction is a much higher temperature reaction than hydrogen reduction. The temperatures are so high that the soil actually becomes molten. Once the melt is established, methane gas is introduced to the reaction chamber. When the methane comes into contact with the high temperature melt, it decomposes into carbon and hydrogen. The carbon is absorbed into the melt where it reacts with some of the minerals and releases carbon monoxide. The carbon monoxide and hydrogen gas are then sent to into a methanation reactor where, in the presence of a catalyst, methane and water are generated. The water is electrolyzed to obtain the oxygen product desired and the hydrogen is sent back into the methanation reactor. This process extracts a much larger percentage of the oxygen available in the regolith, but in introduces the challenge of dealing with molten material.

ORBITEC, of Madison Wisconsin, the contractor who built the Carbothermal system came up with a unique solution to the molten material handling. Instead of using crucibles filled with lunar regolith that would have problems with cleaning, they decided to use the regolith itself as the crucible, since it has very low thermal conductivity. So using a point source of heat it was possible to melt a hemispherical pool of regolith in the center of a bed of regolith. The regolith insulates the reactor walls from the molten material and then when the reaction is complete and the material has solidified it can be simply removed from the bed of regolith, smoothed over, and is complete and the material has solidified

All oxygen production processes have one thing in common, they require significant amounts of thermal energy, and the Carbothermal process is no exception. For the Carbothermal process, resistive heaters were not an attractive option as they would have to come in contact with the molten regolith and the highly reactive environment in the melt would damage the heaters. However, there is another abundant thermal energy source available on the moon, the Sun. It delivers tremendous amounts of thermal energy to the surface of the moon. But collecting it, focusing it and delivering it into the reactor was the challenge that Physical Sciences Incorporated (PSI) tackled through the 
SBIR program. The approach taken utilized parabolic collectors coupled with secondary mirrors to focus the infrared energy into a fiber optic bundle. The fiber optical cable allowed the energy to be routed wherever it was needed. In this case, it was routed to the top of the Carbothermal reactor where the infrared energy was transferred through a quartz rod and focused on the bed of regolith. Though there were losses in the system at each transfer point since all the subsystems were not optimized, more than half of the energy collected is delivered into the chamber, melting the regolith in the reactor in 10 to 15 seconds. The system was even more effective when tested in Hawaii where the test site was 9000 feet above sea level reducing the attenuating effects of the atmosphere.

All of the electrical energy needed to operate the Carbothermal and Solar Concentrator sun tracker were supplied by an air breathing fuel cell. The fuel cell took in the oxygen in the atmosphere reacted it with gaseous hydrogen and produced electricity and water. The water was routed back to the water electrolysis system that was part of the Carbothermal process and regenerated the hydrogen needed by the fuel cell. The oxygen produced by the electrolysis system was routed to a cryogenic storage system where it was liquefied and later used to fire a small methane thruster shown in Figure 3 below.
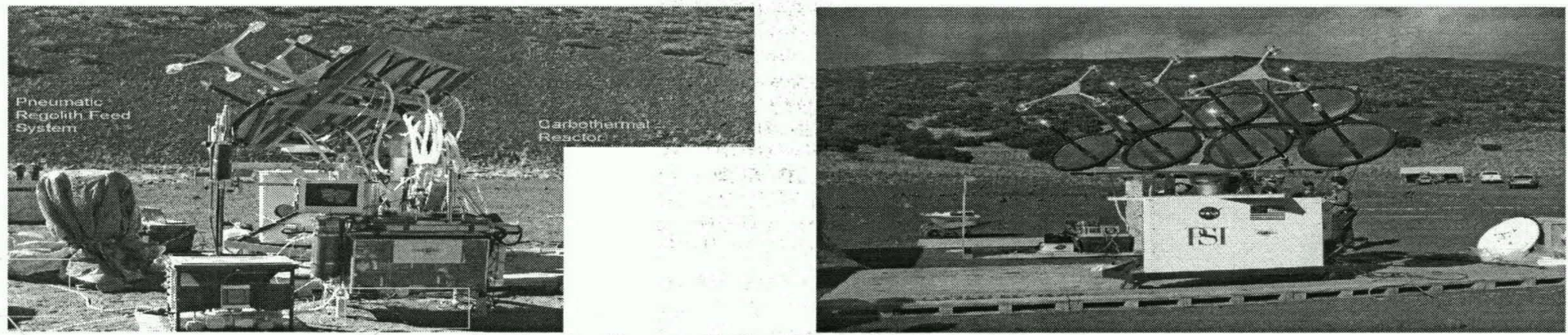

Figure 3: Carbothermal System and Solar Concentrator

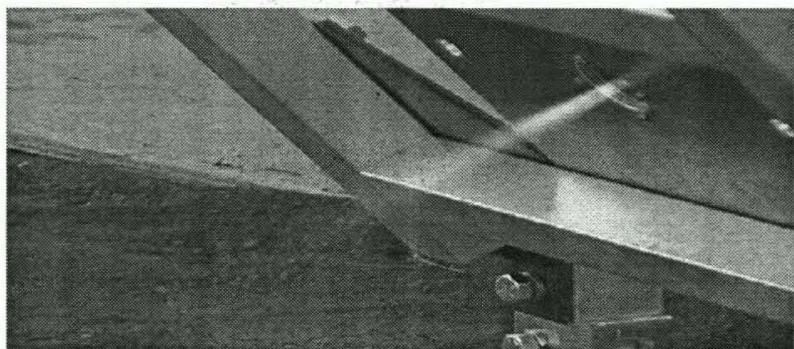

Figure 4: Methane Thruster Firing Using Oxygen Produced From the Soi

\section{B. Lunar Volatiles Prospecting}

Ever since Lunar Prospector detected elevated levels of hydrogen at the lunar poles, there has been considerable debate in the scientific community as to its form and concentration. However, with the impact of LCROSS in the crater Cabeus $A$ in the southern polar region of the moon in October of 2009 , it is clear that there is water ice in the permanently shadowed regions of the poles. Adding to the excitement is Lunar Reconnaissance Orbiter (LRO) data from the Lunar Exploration Neutron Detector that shows elevated regions of hydrogen in areas near the poles that are sunlit for a few days a month. Also, thermal surface temperature maps from the Diviner Lunar Radiometer Experiment onboard LRO support the theory that water ice could be present just 10 's of centimeters below the surface. How much water is in these regions, how is it distributed, and whether this water ice is reasonably accessible as a resource remain an open question. NASA has been designing an mission called RESOLVE

American Institute of Aeronautics and Astronautics 
(Regolith \& Environment Science, and Oxygen \& Lunar Volatile Extraction) to answer many of these questions and more.

RESOLVE will be delivered to the moon on a mobility system so that it can prospect in numerous locations. The first step in the analysis process is to utilize a coring drill to acquire a one meter sample. The core is delivered in segments to a reactor where it is heated slowly to 150 degrees centigrade. As the sample is heated it is monitored by a instruments to detect and quantify the gases being released. In the current version of RESOLVE, a gas chromatograph (GC) is used to analyze the gases. In the flight version of RESOLVE, a mass spectrometer will be added to the exhaust of the GC to provide a more complete analysis of the samples. After the analysis of volatiles is complete RESOLVE uses the reactor to conduct an oxygen production experiment using the hydrogen reduction process. RESOLVE is designed to evaluate a minimum of ten sample sites, though it is believed that it will be capable of many more.

RESOLVE is being developed in partnership with the Canadian Space Agency (CSA), who is providing some of the critical subsystems, like the coring drill and some of the avionics. Ultimately the mission profile for RESOLVE requirement of 200 watts peak, and an experiment mass of 60 kilograms.

RESOLVE has been through two design cycles and has now been tested in the field twice. The initial design cycle was to breadboard all of the subsystems individually and test them to be sure that we were ready to move forward with an integrated design. The next design generation was packaged in a flight-like configuration as shown in the figure below. It was integrated into a rover developed by the Carnegie Mellon University under a grant from NASA's Human Robotics Systems project. The rover which is called Scarab, due to its bug-like appearance, was designed specifically to carry the Canadian-supplied drill system and the volatiles analysis system. The integrated hardware is shown at the 2008 ISRU field test at a lunar analog test site in Hawaii provided by the Pacific International Space Center for Exploration Systems (PISCES). The field test showed that RESOLVE could accomplish its design goals. But the constrained timeframe of the field test left the team with a design for more test time.
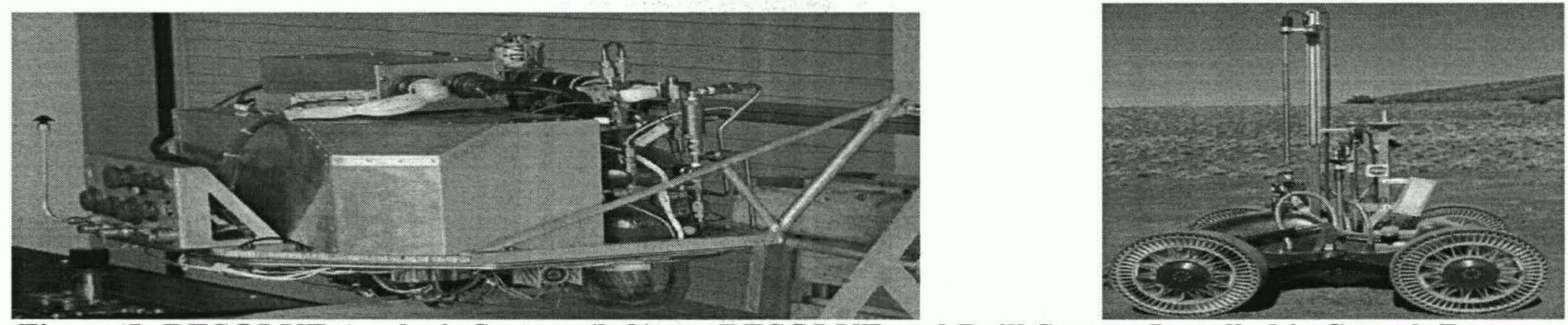

Figure 5: RESOLVE Analysis System (left)

RESOLVE and Drill System Installed in Scarab Rover

In 2010 the RESOLVE team was able to return the hardware to the field under a award from the Science Mission Directorate. This time the CSA supplied the mobility platform for RESOLVE. In the timeframe between the field tests, the design team focused on reducing the size of the power and control systems. Due to this miniaturization, RESOLVE was able to rove into the bottom of a volcanic cinder cone where it collected samples and analyzed the soil, simulating one of the possible scenarios for a lunar mission. As a result of these two tests the team is ready to move forward with a flight design.

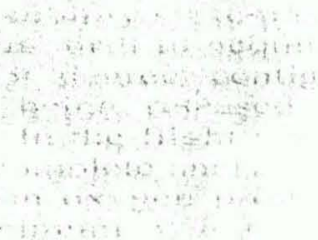




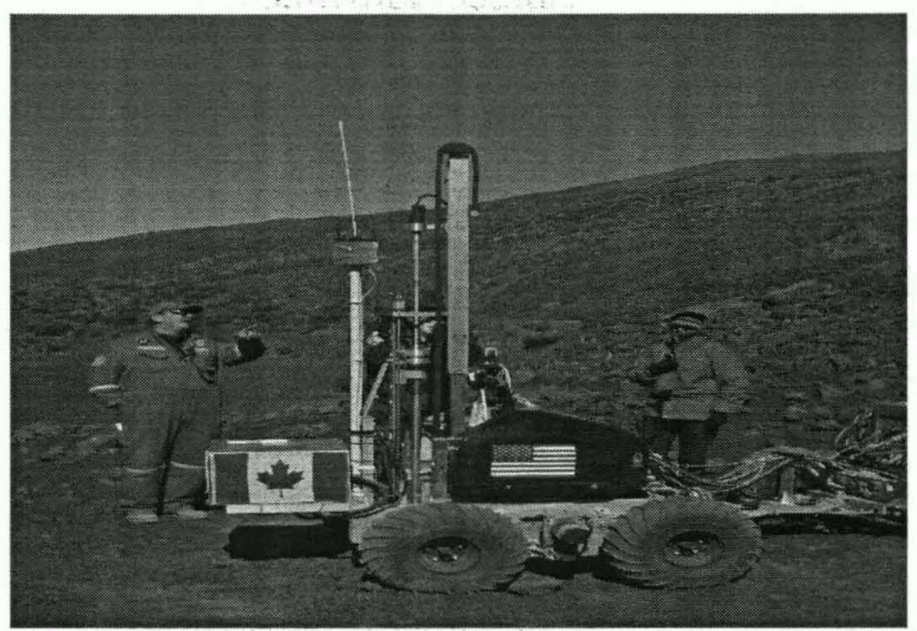

Figure 6: RESOLVE and CSA Rover at 2010 Field Test

\section{The ISRU Project Going Forward}

Under the new Exploration Enterprise as agreed to by the President and Congress, the ISRU project's scope expands once again to include many destinations and a broad range of technologies. While the current thoughts on a human NEO mission would not require ISRU at the destination, propellants derived from the moon could ultimately support missions beyond earth orbit. Therefore, the project will continue some of its lunar work while we begin to include work related to deep space missions, specifically Mars.

Not only has NASA's mission focus changed with the new Exploration Enterprise, but the technology program going forward will be quite different from the current program. There will be an increased focus on external participation and multiple sources of funding. It will also allow for the scope of the ISRU project to be broadened beyond oxygen production from lunar regolith to the use of space resources to provide fuel, water, metals, plastics and even the manufacture of spare parts.

The new technology development structure within NASA will have several programs that could potentially fund ISRU activities. One of the programs, Enabling Technology Development and Demonstration (ETDD), will be the home of the ISRU Domain. There is an additional program called the Space Technology Program that is managed by the Office of the Chief Technologist that is also likely to fund low TRL ISRU development. This program is intended to fund technologies that cross cut NASA's missions and can be "game changing." The majority of the technologies and systems development under the Space Technology Program will be awarded competitively allowing greater opportunity for industry and academia to contribute to NASA's development efforts.

The ETDD program will provide Domain funding to allow NASA to maintain its core technical expertise. The Domain will provide the technical leadership; establishing the technology development strategy and perform the system integration and development activities for ISRU. The ETDD program will also have three additional elements, Demonstrations, Skunk works and a Competitive Technology Development element. Each Demonstration Project is framed around answering a key question derived from architectural studies. Demonstration Project Managers, who are separate from the Domain Managers, competitively select technologies to demonstrate solutions to those key questions. It is expected that technologies from several Foundational Technology Domains will be chosen for inclusion in each of the Demonstrations. Skunk works projects will be established as a way to utilize inhouse civil service labor to tackle technology developments that will enhance NASA's ability to conduct exploration missions. Finally, the Competitive Technology Development element will utilize Broad Area Announcements to solicit technology development projects from Academia and Industry insuring that new approaches to ISRU receive an opportunity to mature.

In Fiscal Year the ISRU Domain will continue to develop some lunar related technologies and begin work on some Mars and multidestinational technologies. RESOLVE will start down the path of designing for a flight to the 
moon to provide ground truth for the lunar water. Mission architecture trade studies are already underway as is design work to build flight electronics and flight certifiable software. We also will redesign the reactor to improve its seals. The flight schedule for RESOLVE is still uncertain, but there appear to be several realistic opportunities to Multidestinational work such as producing pure metals from planetary regolith and trash processing to produce fuel or water will continue. We will also initiate a new project to begin testing concepts for the extraction of water from planetary regolith since there now appears to be abundant water on both Mars and the moon. We will also initiate several trade studies to help us understand the either the current state of the art in a technology area or the viability of an ISRU-based architecture.

Fiscal year 2012 will bring the beginning of the external technology development segment of ETDD. The budget for the ETDD competitive program is somewhat constrained initially, but is slated to grow over the next five years. Using the results of the State of the Art studies conducted internally in FY11, the ISRU Domain will craft a section of the Broad Area Announcement that the ETDD program plans to issue that will cover all of the ETDD technology domains. The competitions between NASA technologists and external providers will be conducted separately. However, for the external competitions, partnerships will be encouraged between the external researchers and a NASA Center. Subject to budget availability it is hoped that this externally awarded element of the ETDD Program will grow to $50 \%$ of our funding.

\section{Conclusion}

The ISRU project has made tremendous strides over the last five year, and the future promises more progress. While there remains some uncertainty regarding the funding levels available due to negotiations still underway in Congress, there is a interest in ISRU from the language of the Authorization Act. So the ISRU project should be able to execute our plans to advance the state of the art and to include academia and industry in our efforts.

\section{Acknowledgments}

The authors would like to acknowledge the many fine men and women that work on the ISRU project. Their dedication and capabilities have allowed ISRU to make tremendous strides in just a short amount of time.

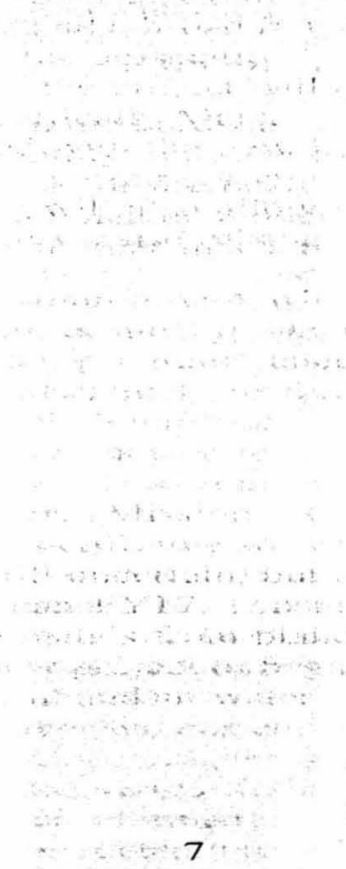

